\title{
Editorial
}

\section{European Symposium on Friction, Wear, and Wear Protection}

\author{
Martin Dienwiebel, ${ }^{1,2}$ Matthias Scherge, ${ }^{1}$ Michael Moseler, ${ }^{1,3}$ Alfons Fischer, \\ Ken'ichi Hiratsuka, ${ }^{5}$ and Julien Fontaine ${ }^{6}$ \\ ${ }^{1}$ Fraunhofer Institute for Mechanics of Materials (IWM)-Microtribology Center $\mu$ TC, Wöhlerstrasse 11, 79108 Freiburg, Germany \\ ${ }^{2}$ Karlsruhe Institute of Technology KIT, Institute for Applied Materials IAM, Kaiserstrasse 12, 76131 Karlsruhe, Germany \\ ${ }^{3}$ Institute of Physics, Albert-Ludwigs-University Freiburg, Hermann-Herder-Straße 3a, 79104 Freiburg, Germany \\ ${ }^{4}$ Institute of Product Engineering, University Duisburg-Essen, Lotharstraße 1, 47057 Duisburg, Germany \\ ${ }^{5}$ Chiba Institute of Technology, 2-17-1 Tsudanuma, Narashino-shi, 275-8588 Chiba, Japan \\ ${ }^{6}$ Laboratoire de Tribologie et Dynamique des Systèmes, UMR 5513 CNRS, Ecole Centrale de Lyon, Université de Lyon, \\ 36 Avenue Guy de Collongue, 69134 Ecully Cedex, France
}

Correspondence should be addressed to Martin Dienwiebel; martin.dienwiebel@kit.edu

Received 1 March 2015; Accepted 1 March 2015

Copyright (C) 2015 Martin Dienwiebel et al. This is an open access article distributed under the Creative Commons Attribution License, which permits unrestricted use, distribution, and reproduction in any medium, provided the original work is properly cited.

The 3rd European Symposium on Friction, Wear, and Wear Protection was held in Karlsruhe from May 6 to May 8, 2014, under the auspices of the German Materials Research Society DGM. Since conservation of energy and resources is one of the biggest challenges for society and industry, sharing and expanding knowledge in tribology is an important cornerstone to reach that goal.

In that respect, with almost 200 delegates from 26 countries and 5 continents attending the conference, it was a great success and underpinned the organizers' intention that the symposium would provide a forum for exchange of the International Tribology Community with German academia and industry. The scope of the conference has expanded and ranged from wear and friction phenomena, modeling and simulation, and friction materials in brakes and clutches to implant- and nanotribology. Materials ranging from ceramics and coating to polymers and PMCs were addressed in dedicated sessions. This special issue features nine papers that cover this range of materials and deal with the erosion of coatings, the wear resistance of alloys and composites, the effect of microstructuring of surfaces, the oxidation of tribological contacts, and the development of new tribometers to investigate tool wear. With this ongoing interest and rising participation the successful continuation of the symposium series beyond 2014 is guaranteed.

\section{Acknowledgments}

On behalf of the conference chairmen, I would like to thank the Scientific Programme Committee for their contribution in selecting the most interesting and novel abstracts. I also like to thank all plenary speakers (Dr. Somuri Prasad, Professor Markus Wimmer, Professor Staffan Jacobson, and Professor Dirk Bartel) who started the first day of the symposium with insightful overview lectures. Furthermore, I thank all speakers and poster presenters for sharing their latest research and interesting discussions. Finally I would like to express my gratitude to the staff of the DGM (Anja Mangold and Kristin Tonn) for the perfect organization of the meeting.
Martin Dienwiebel Matthias Scherge Michael Moseler Alfons Fischer Ken'ichi Hiratsuka Julien Fontaine 

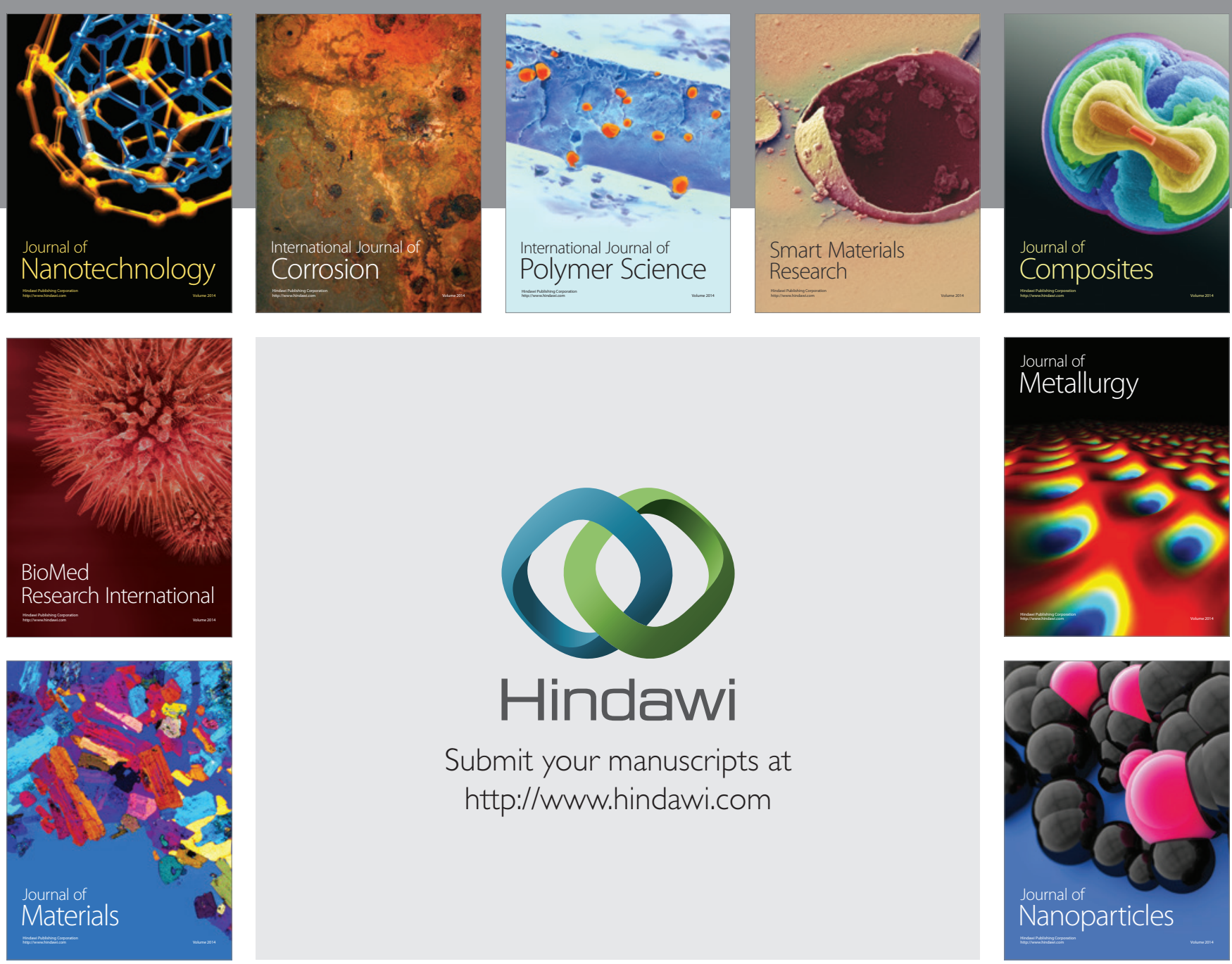

Submit your manuscripts at http://www.hindawi.com
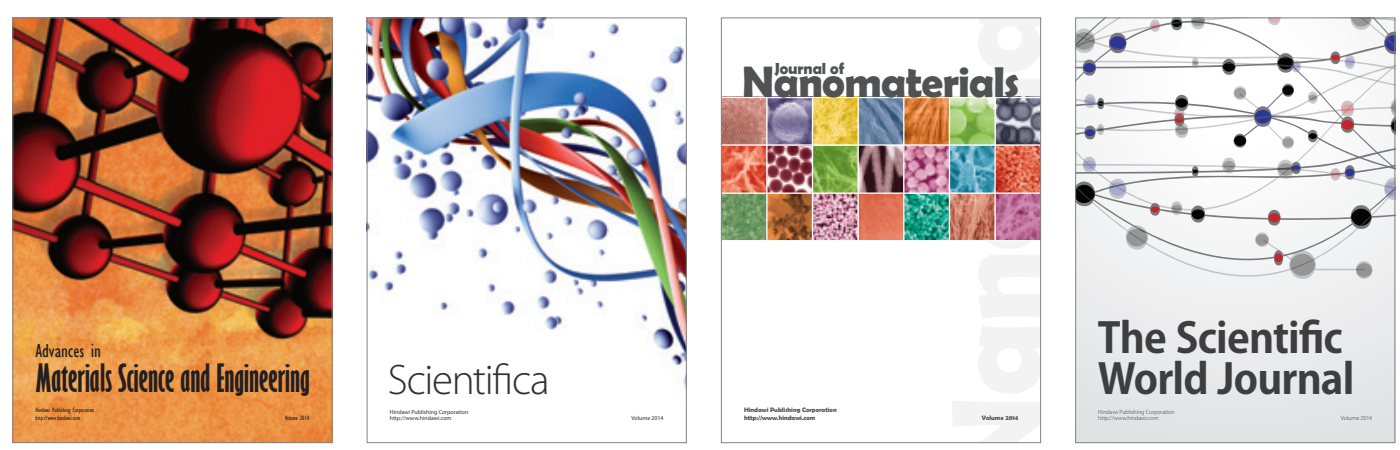

\section{The Scientific World Journal}
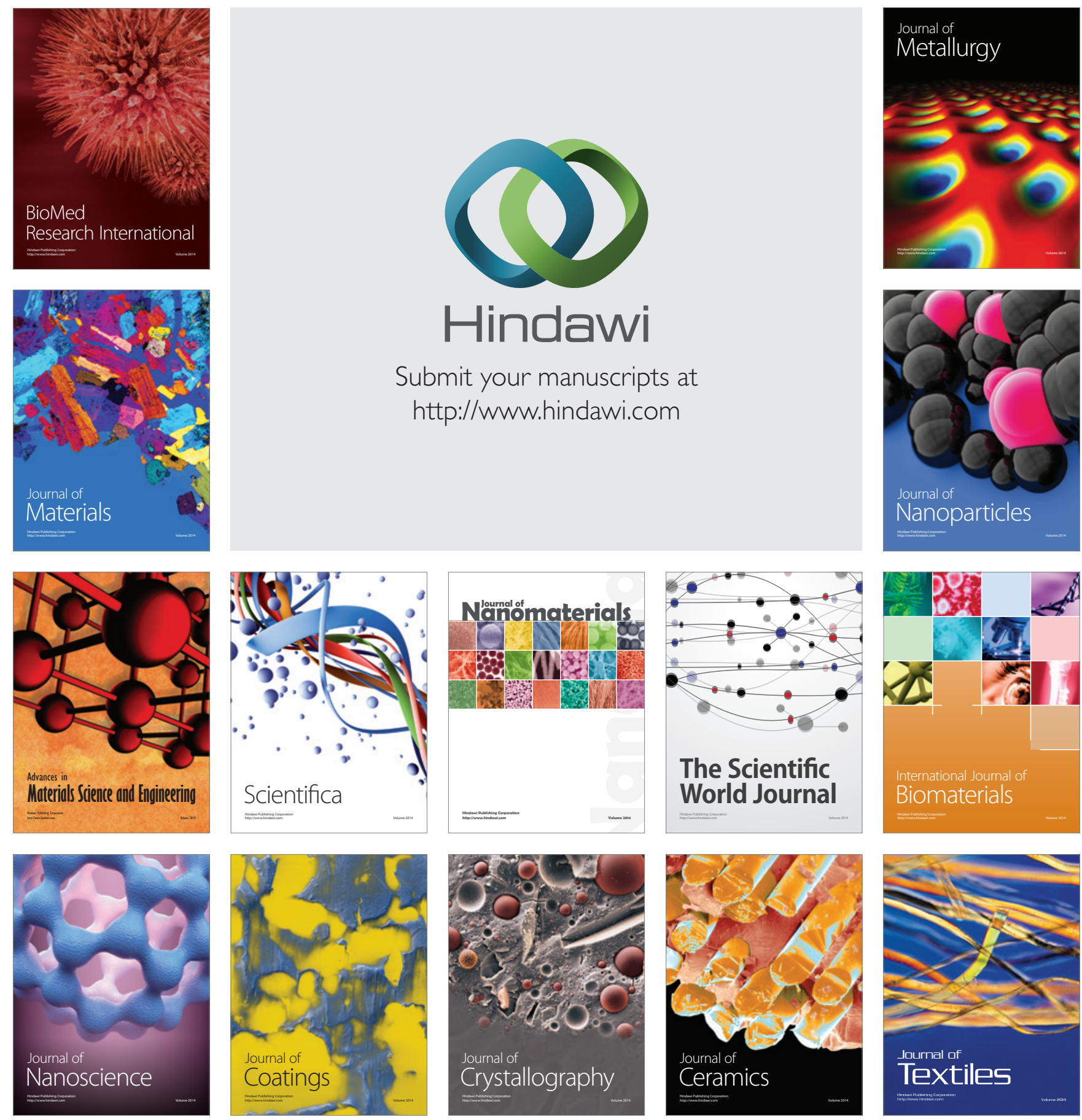